\title{
Estrés postraumático en escolares a 8 meses del $27 \mathrm{~F}$
}

\author{
ANA M. BRICEÑO A. ${ }^{1}$, MARCELA ABUFHELE M. ${ }^{1}$, ANA MARÍA DÁVILA D. ${ }^{2}$, \\ MICHELLE BARREAU V. ${ }^{1}$, KATERINA SOMMER A. ${ }^{1}$, SONIA CASTRO M. ${ }^{2}$, \\ CAROLINA EBEL F. ${ }^{2}$, SANDRA OLTRA H. ${ }^{2}$, MARCELA ARAVENA P. ${ }^{1}$, \\ CLAUDIO MELO A. ${ }^{1}$, ALFONSO CORREA D. ${ }^{1}$ \\ 1. Psiquiatras Infantiles, Unidad Psiquiatría Infantil-Departamento de Pediatría. Clínica Alemana de Santiago, \\ Facultad de Medicina Clínica Alemana, Universidad del Desarrollo, Santiago, Chile. \\ 2. Psicóloga, Unidad Psiquiatría Infantil-Departamento de Pediatría. Clínica Alemana de Santiago, Facultad de Medicina \\ Clínica Alemana, Universidad del Desarrollo, Santiago, Chile.
}

\begin{abstract}
Post-traumatic stress disorder in children eight months after february 27, 2010

Introduction: There are several epidemiological studies regarding the incidence of post-traumatic stress disorder (PTSD) in children exposed to natural disasters. Objective: To describe the prevalence of PTSD in a school-age population in a coastal town from the Maule Region, 8 months after the earthquake/tsunami in February 2010, and to compare differences among PTSD groups of symptom (re-experiencing, avoidance and activation) according to demographic variables such as age, grade, gender and family type. Methodology: The Child PTSD Symptom Scale (CPSS), validated in Chile in 2009, was used in 89 children between 3rd and 6th grade, corresponding to $94.7 \%$ of the children enrolled in the local school in such classes. Data are analyzed by gender, age, grade and type of family. 89 surveys were applied, $59.6 \%$ were male and $40.4 \%$ female aged 8-13. Results: $40.4 \%$ of children had symptoms consistent with PTSD, with higher incidence in women and younger children; the most significant association among women was age. Both groups (women and young children) presented the highest scores on all group of symptoms. There were no differences by type of family. Conclusions: The incidence of PTSD measured by CPSS scale in the study population was $40.4 \%$, considered to be among the highest percentages reported in the international literature.
\end{abstract}

(Key words: Post-traumatic stress disorder (PTSD), epidemiology, earthquake).

Rev Chil Pediatr 2013; 84 (1): 42-50

Recibido el 13 de agosto de 2012, devuelto para corregir el 3 de octubre de 2012, segunda versión el 6 de noviembre de 2012 , aceptado para publicación el 3 de diciembre de 2012.

Financiamiento: Clínica Alemana de Santiago (traslados, viático), Laboratorio Andrómaco (apoyo alimentación), Tiempo Protegido Clínica Alemana de Santiago (redacción manuscrito).

Este trabajo cumple con los requisitos sobre consentimiento /asentimiento informado, comité de ética, financiamiento, estudios animales y sobre la ausencia de conflictos de intereses según corresponda.

Correspondencia a:

Ana Marina Briceño Arias

E-mail: anambriceno@gmail.com 


\section{RESUMEN}

Introducción: Existen diversos datos epidemiológicos respecto a la incidencia de Trastorno por Estrés Postraumático (TEPT) en niños expuestos a desastres naturales. Objetivo: Describir la prevalencia de TEPT en una población infantil escolarizada de una localidad costera de la Región del Maule después de 8 meses de ocurrido el terremoto/maremoto de febrero/2010, y comparar las diferencias entre grupos de síntomas del TEPT (reexperimentación, evitación y activación) según variables demográficas, como edad, curso, sexo y tipo de familia. Metodología: Se aplicó la escala Child PTSD Symptom Scale (CPSS) validada en Chile el año 2009 , a 89 niños de $3^{\circ}$ a $6^{\circ}$ básico lo que corresponde al 94,7\% de los niños matriculados en la escuela de la localidad en dichos cursos. Se analizan los datos según sexo, edad, curso y tipo de familia. Se aplicaron 89 encuestas, 59,6\% eran varones y 40,4\% mujeres de 8 a 13 años de edad. Resultados: El 40,4\% del total de niños tuvo una evaluación compatible con TEPT, con mayor incidencia en mujeres y niños de menor edad, siendo la edad un factor de asociación significativa en las mujeres. Ambos grupos (mujeres y niños más pequeños) presentaron mayores puntajes en todos los grupos sintomáticos. No se encontraron diferencias según el tipo de familia. Conclusiones: La incidencia de TEPT medida a través de la escala CPSS en la población estudiada fue de $40.4 \%$, encontrándose entre las más altas reportadas en la bibliografía internacional.

(Palabras clave: Trastorno por estrés postraumático (TEPT), epidemiología, terremoto).

Rev Chil Pediatr 2013; 84 (1): 42-50

\section{Introducción}

En la madrugada del 27 de febrero de 2010, un terremoto de 8,8 grados en la escala de Richter afectó al centro/sur de Chile, siendo catalogado dentro de los 8 más grandes registrados en el mundo'. El epicentro ocurrió en el mar, al noroeste de Concepción, la segunda ciudad más grande del país. A los pocos minutos del terremoto, las localidades costeras cercanas al epicentro fueron impactadas por un maremoto ${ }^{2}$.

En el marco del convenio de colaboración para apoyo en salud integral entre Clínica Alemana de Santiago y una Municipalidad de la Región del Maule, se organizó un programa psicológico y psiquiátrico de 10 meses de duración de atención de niños, niñas y adolescentes y apoyo al profesorado, cuyo diseño e implementación se describen en otras publicaciones de estos autores ${ }^{3,4}$.

Para los niños, al igual que los adultos, los efectos psicológicos que se producen posterior a desastres naturales son muy variados, la mayoría de los cuales cumple una función inicialmente adaptativa, sin implicar patología necesariamente ${ }^{5}$. No obstante, también se describe la aparición de respuestas patológicas en un porcentaje de la población, destacando entre ellos trastornos del ánimo, trastornos por estrés postraumático (TEPT), trastornos de pánico y fobias específicas ${ }^{6-8}$.

Dentro del amplio espectro de respuestas adaptativas y patológicas al estrés, un cuadro de especial severidad es el TEPT, pues provoca malestar clínico o deterioro en áreas importantes de la actividad del individuo, que en el caso de los niños implica alteraciones en su desarrollo psicosocial y aumento del riesgo de psicopatología a futuro ${ }^{7,9,10}$. El TEPT se define por la aparición de síntomas característicos que siguen a la exposición de un acontecimiento estresante y traumático, donde el individuo se ve envuelto en hechos que representan un peligro real para su vida, integridad física o es testigo de un acontecimiento donde existe una amenaza para la vida de otras personas ${ }^{6}$.

La reacción emocional experimentada implica una respuesta de temor, desesperanza $u$ horror intensos, que se prolonga por más de un mes desde el suceso traumático. Se describen tres grupos de síntomas: re-experimentación del acontecimiento traumático, evitación de estímulos asociados al trauma o embotamiento y aumento de la activación ${ }^{6}$. Los síntomas de reexperimentación son los que se han descrito como más invalidantes y asociados a peores pronósticos en la adolescencia y adultez, tales como autoagresiones y consumo problemático de alcohol, dando mayor severidad al cuadro 
de TEPT ${ }^{11-14}$. No obstante, recientemente se ha cuestionado la organización de estos criterios en niños y adolescentes, sugiriendo reordenarlos en próximos manuales diagnósticos ${ }^{15}$.

Estudios sobre el efecto psicológico de los terremotos en niños, revelan una gran variación en las tasas de TEPT, que van desde 4,5\% a 95\%, dependiendo de la intensidad, duración y proximidad al acontecimiento traumático; influirían además de características individuales del niño y su entorno y las escalas utilizadas, entre otras variables ${ }^{16-19}$. El tiempo pasado entre el evento traumático y la medición también es relevante, pues es esperable que la incidencia poblacional del cuadro vaya decreciendo ${ }^{19-21}$.

El riesgo de desarrollar TEPT se ha visto relacionado con múltiples factores, tales como sexo femenino, menor edad, la cercanía a la zona afectada y el tipo de desastre ${ }^{22-24}$; los terremotos están dentro de los desastres naturales más atemorizantes, debido al impacto que generan por su grado de devastación ${ }^{19}$. Respecto a la edad, hay evidencia contradictoria, pues aunque la mayoría plantea que tener menor edad es un factor asociado a mayor TEPT, hay estudios que han encontrado lo contrario ${ }^{25}$. Otras características demográficas no se han relacionado con cambios en la incidencia, tales como tipo de familia, educación de los padres o religión ${ }^{26}$. En un artículo ${ }^{25}$, Liu y cols., plantean que además de analizar la edad de los niños, es relevante estudiar la incidencia de TEPT por curso, considerando que el nivel académico daría cuenta del desarrollo cognitivo de los niños. Respecto al tipo de familia, no se ha encontrado hasta el momento relación entre síntomas de TEPT con la composición familiar ${ }^{26}$, pero sí se ha evidenciado la relación entre TEPT infantil y síntomas de TEPT en los adultos relacionados con el niño ${ }^{27}$.

El objetivo del presente trabajo es describir la prevalencia de TEPT en una población infantil escolarizada de una localidad costera, medido con la escala CPSS después de 8 meses de ocurrido el terremoto/maremoto de 2010, y comparar las diferencias entre grupos de síntomas del TEPT (reexperimentación, evitación y activación) según variables demográficas, como edad, curso, sexo y tipo de familia.

\section{Participantes y Método}

Se evaluó una muestra por conveniencia, de un total de 89 niños luego de 8 meses de ocurrido el terremoto/maremoto, que correspondió al 94,7\% de los niños matriculados en el colegio de la localidad (el único de educación básica que estaba operativo) entre $3^{\circ}$ y $6^{\circ}$ básico. Se escogieron estos cursos debido a la edad de validación del instrumento y a que $6^{\circ}$ básico es el mayor curso del establecimiento. Cinco niños no se pudieron encuestar debido a su inasistencia a clases durante la evaluación. Del total de niños evaluados, el 40,4\% corresponde a mujeres y el $59,6 \%$ a varones, con un promedio de edad de 9,8 años (rango entre 8 y 13 años). Es importante destacar que de los 89 niños entrevistados, un niño no rellenó su edad, por lo que fue excluido en los análisis que se refieren a este dato.

\section{Procedimiento e instrumento}

Se aplicó la Escala Infantil de Síntomas del Trastorno de Estrés Postraumático (CPSS), desarrollada por Foa et $\mathrm{al}^{28}$ y validada en Chile por Bustos et $\mathrm{al}^{7}$, con adecuadas propiedades psicométricas. Fue diseñada para evaluar la presencia y severidad de síntomas de TEPT en niños/as y adolescentes de 8 a 18 años con una historia conocida de trauma y está basada en los criterios diagnósticos del DSM-IV. Se compone de 3 sub-escalas, relacionadas con los siguientes grupos sintomáticos: re-experimentación ( 5 ítems), evitación ( 7 ítems) y aumento de la activación (5 ítems), sumando un total de 17 ítems; con respuestas tipo Likert referidos a la frecuencia de manifestación de síntomas de estrés post-traumáticos en las últimas dos semanas (0: nunca, 1: sólo una vez, 2: entre 2 y 3 veces, 3 : entre 4 y 8 veces y $4: 9$ veces o más). La versión chilena de la CPSS se validó preliminarmente en niños entre 8 y 18 años, y se mostró que discrimina significativamente entre quienes presentan un TEPT alto (puntuaciones de sintomatología TEPT moderada o alta) o TEPT bajo (puntuaciones de sintomatología TEPT nula o baja), siendo el puntaje de corte de 24 puntos. Tiene un rango de puntaje entre 0 y 68 puntos y además permite diferenciar los puntajes de las diferentes subes- 
calas. Junto con la escala, se pidió a los niños que escribieran un listado de las personas con quienes vivían, para identificar la estructura familiar, diferenciándose luego como familia nuclear (con ambos padres), nuclear extendida (con ambos padres y algún otro adulto), monoparental (sólo uno de los padres), monoparental extendida (uno de los padres y otro adulto) y familia sin padres (vive con familiares, pero sin sus padres).

Se realizó una aplicación grupal asistida en la sala de clases por psiquiatras y psicólogos, acompañados por la profesora jefe del curso. Dada la aplicación en el contexto de una intervención global aprobada por la comunidad escolar y la Municipalidad, se recibió sólo el consentimiento verbal de la directora del colegio, profesores, apoderados y niños. Los niños que puntuaron sobre el punto de corte fueron informados y derivados a atención psicológica y/o psiquiátrica. La descripción de la intervención se encuentra en otros artículos de los autores $^{3,4}$; esta evaluación se realizó casi al finalizar el proceso de intervención que se describe en dichos trabajos (lo que explica la elección del período de 8 meses post-terremoto).

\section{Análisis estadístico}

Para las variables categóricas o discretas se utilizaron tablas de contingencia y $\chi^{2}$ de Pearson o Test exacto de Fischer. Para las variables cuantitativas se utilizó análisis de varianzas (ANOVA). Para todos los análisis se utilizó el software SPSS versión 19.0, con excepción del análisis estadístico de los diferentes estudios (tabla 6), en que se aplicó Test de $\chi^{2}$ de Pearson con tablas de contingencia, utilizando el software Epidat 3.1.

\section{Resultados}

El puntaje promedio de la escala CPSS en esta muestra fue de 22,66 puntos, una desviación estándar (D.S.) de 16.174 y un rango de puntaje entre 1 y 61 puntos.

Un $40,4 \%$ de los niños encuestados puntuaron sobre el punto de corte de la escala, dando positivos un $51,4 \%$ de las mujeres y un $34 \%$ de los hombres $(\mathrm{p}=0,05)$. El análisis de puntaje por edad se muestra en la tabla 1 , con diferencias significativas, que muestran una tendencia descendiente en puntaje al ir aumentando la edad. El análisis dicotómico (TEPT sí/no) por edad y sexo se aprecia en la tabla 2, donde se aprecia que existen diferencias por edad en ambos sexos, con mayor prevalencia a menor edad; sin embargo, éstas diferencias son significativas sólo en mujeres.

En la figura 1 se muestran los resultados por curso, llamando la atención la prevalencia significativamente mayor en $3^{\circ}$ básico $(p=0,003)$.

Al separar las subescalas que corresponden a los diferentes grupos sintomáticos, se obser-

Tabla 1. Análisis de puntaje CPSS por edad

\begin{tabular}{|ccc|}
\hline Edad (n) & $\overline{\mathbf{x}}$ & DS \\
\hline $8(06)$ & 43,67 & 9,37 \\
$9(26)$ & 26,42 & 16,61 \\
$10(22)$ & 15,95 & 12,47 \\
$11(20)$ & 16,75 & 14,52 \\
$12(10)$ & 25,50 & 15,60 \\
$13(04)$ & 18,00 & 10,07 \\
Significancia & \multicolumn{3}{|c}{$p=0,001$} \\
\hline Test ANOVA gl=87.
\end{tabular}

Tabla 2. TEPT según sexo y edad

\begin{tabular}{|c|c|c|c|c|c|c|}
\hline \multicolumn{3}{|c|}{ Sexo } & \multicolumn{2}{|c|}{ Puntaje CPSS } & \multirow[t]{2}{*}{$\mathbf{n}$} & \multirow[t]{2}{*}{$\mathbf{p}$} \\
\hline & & & $<24$ & $\geq 24$ & & \\
\hline \multirow[t]{6}{*}{$F$} & Edad & 8 & 0 & 4 & 4 & \\
\hline & & 9 & 2 & 7 & 9 & \\
\hline & & 10 & 7 & 2 & 9 & \\
\hline & & 11 & 8 & 1 & 9 & \\
\hline & & 12 & 1 & 4 & 5 & \\
\hline & Total & & 18 & 18 & 36 & 0,002 \\
\hline \multirow[t]{7}{*}{ M } & Edad & 8 & 0 & 2 & 2 & \\
\hline & & 9 & 10 & 7 & 17 & \\
\hline & & 10 & 11 & 2 & 13 & \\
\hline & & 11 & 6 & 5 & 11 & \\
\hline & & 12 & 5 & 0 & 5 & \\
\hline & & 13 & 3 & 1 & 4 & \\
\hline & Total & & 35 & 17 & 52 & 0,081 \\
\hline
\end{tabular}

Test $\chi^{2}$ gl fem: 4 //gl masc: 5 . 


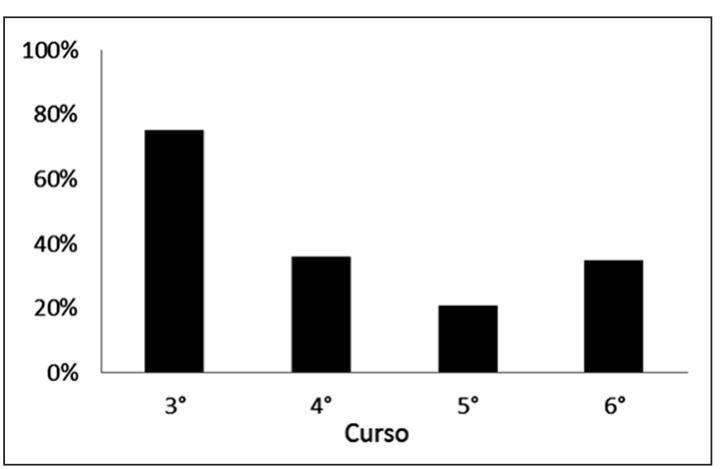

Figura 1. Prevalencia TEPT según curso. va una significativa mayor frecuencia de síntomas de evitación y activación en mujeres (tabla 3). A menor edad, hay significativamente mayor puntaje en las tres subescalas (tabla 4).

En la tabla 5 se aprecian los puntajes de CPSS subdivididos por subescalas según el tipo de familia, sin mostrar diferencias significativas. Sin embargo, llama la atención la tendencia de menores puntajes en familias en teoría más vulnerables (monoparentales o quienes no viven con sus padres).

Tabla 3. Diferencias entre grupos de síntomas según sexo

\begin{tabular}{|lcccccc|}
\hline Sexo (n) & \multicolumn{2}{c}{ Reexperim } & \multicolumn{2}{c}{ Evitación } & \multicolumn{2}{c|}{ Activación } \\
& $\overline{\mathbf{x}}$ & DS & $\overline{\mathbf{x}}$ & DS & $\overline{\mathbf{x}}$ & DS \\
Niñas (36) & 8,278 & 6,273 & 9,556 & 7,319 & 9,361 & 6,234 \\
Niños (53) & 6,623 & 4,797 & 6,113 & 5,639 & 6,849 & 5,149 \\
Significancia & \multicolumn{2}{c}{$\mathrm{p}=0,162$} & \multicolumn{2}{c}{$\mathrm{p}=0,014$} & & $\mathrm{p}=0,041$ \\
\hline
\end{tabular}

Test ANOVA gl=89.

Tabla 4. Diferencias entre grupos de síntomas según edad

\begin{tabular}{|c|c|c|c|c|c|c|}
\hline \multirow[t]{2}{*}{ Edad (n) } & \multicolumn{2}{|c|}{ Reexperim } & \multicolumn{2}{|c|}{ Evitación } & \multicolumn{2}{|c|}{ Activación } \\
\hline & $\overline{\mathbf{x}}$ & DS & $\overline{\mathbf{x}}$ & DS & $\overline{\mathbf{x}}$ & DS \\
\hline 8 (6) & 13,17 & 5,382 & 17,00 & 3,464 & 13,50 & 2,881 \\
\hline $9(26)$ & 8,92 & 5,291 & 8,81 & 6,859 & 8,69 & 5,563 \\
\hline $10(22)$ & 6,36 & 5,132 & 4,36 & 4,249 & 5,23 & 5,070 \\
\hline $11(20)$ & 5,30 & 4,868 & 5,70 & 6,284 & 5,75 & 5,118 \\
\hline $12(10)$ & 6,00 & 4,422 & 8,60 & 6,433 & 10,90 & 5,744 \\
\hline $13(4)$ & 3,25 & 2,217 & 6,25 & 5,377 & 8,50 & 3,317 \\
\hline Total & 7,16 & 5,354 & 7,41 & 6,530 & 7,73 & 5,597 \\
\hline Significancia & \multicolumn{2}{|c|}{$p=0,005$} & \multicolumn{2}{|c|}{$p=0,0001$} & \multicolumn{2}{|c|}{$p=0,002$} \\
\hline
\end{tabular}

Test ANOVA gl $=88$.

Tabla 5. Puntaje CPSS según tipo de familia

\begin{tabular}{|lcccc|}
\hline Tipo familia $(\mathbf{n})$ & Reexperim & Evitación & Activación & CPSS total \\
\hline Nuclear (51) & 7,80 & 8,49 & 8,31 & 24,61 \\
\hline Nuclear extendida (10) & 7,40 & 6,80 & 8,00 & 22,20 \\
\hline Monoparental (8) & 6,75 & 4,50 & 5,13 & 16,38 \\
Monoparental extendida (13) & 6,69 & 7,92 & 9,23 & 23,85 \\
\hline Familia sin padres (7) & 5,14 & 4,00 & 5,00 & 14,14 \\
\hline Total (89) & 7,29 & 7,51 & 7,87 & 22,66 \\
\hline Significancia & $p=0,786$ & $p=0,290$ & $p=0,328$ & $p=0,417$ \\
\hline
\end{tabular}

Test ANOVA $g l=88$. 
Tabla 6. Comparación prevalencia de TEPT en Chile según edad

\begin{tabular}{|c|c|c|c|c|c|c|c|}
\hline \multicolumn{2}{|c|}{ Estudios/prevalencia TEPT } & \multicolumn{2}{|c|}{$\begin{array}{c}\text { Prevalencia > } 15 \text { años } \\
\text { (Pérez-Benítez et al) } \\
\text { (A) }\end{array}$} & \multicolumn{2}{|c|}{$\begin{array}{l}\text { 13-19 años } \\
\text { Terremoto/maremoto } \\
\text { (Díaz et al. 2012) } \\
\text { (B) }\end{array}$} & \multicolumn{2}{|c|}{$\begin{array}{c}\text { 8-13 años } \\
\text { Terremoto/maremoto } \\
\text { (C) }\end{array}$} \\
\hline Hombres & $(n)$ & $2,5 \%$ & $(1245)$ & $7,8 \%$ & $(128)$ & $34 \%$ & $(53)$ \\
\hline Mujeres & $(n)$ & $6,2 \%$ & $(1358)$ & $29,5 \%$ & $(176)$ & $51,4 \%$ & (36) \\
\hline Total & (n) & $8,7 \%$ & (2 603) & $20,4 \%$ & $(304)$ & $40,4 \%$ & (89) \\
\hline \multicolumn{2}{|c|}{ Comparación con trabajo C } & \multicolumn{2}{|c|}{$p=0,001$} & \multicolumn{2}{|c|}{$p=0,0001$} & & \\
\hline
\end{tabular}

Test $\chi^{2}$.

\section{Discusión}

Dentro de las consecuencias de los desastres naturales en la salud mental, los que se producen en los niños son de gran importancia, debido a que se considera una población de alta vulnerabilidad a psicopatología. El TEPT es una de las patologías de mayor gravedad, dado que puede interferir en forma significativa en las actividades cotidianas de los afectados. Este estudio tuvo como objetivo conocer la incidencia de síntomas de TEPT en niños de una localidad afectada por un maremoto/ terremoto, y estudiar su relación con variables demográficas, como edad, sexo, curso y tipo de familia, además de describir los grupos de síntomas de TEPT más frecuentes en la población estudiada.

La prevalencia de TEPT en la población en estudio fue de $40,4 \%$, superando muchos reportes de la literatura, en que fluctúan desde $4,5 \%$ hasta un $74,5 \%{ }^{19}$ de los niños expuestos. Si se compara con desastres similares y estudios realizados a tiempos equivalentes del suceso ( 8 meses), la prevalencia que muestra este estudio es mayor a la encontrada en población de la misma edad en China, Turquía y Grecia ${ }^{19,25,29}$ y sólo similar a la encontrada en niños sobrevivientes del terremoto en Armenia de $1988^{30}$, uno de los más devastadores de la historia, y que tuvo la particularidad de que los niños se encontraban en clases durante su ocurrencia. Esta alta prevalencia se podría explicar por la magnitud del desastre, y por la reacción de los adultos cuidadores, que se han evidenciado en la literatura como factores que se asocian al TEPT en niños ${ }^{31,32}$.
En Chile, aún no hay publicaciones de efectos en salud mental del terremoto/maremoto del 27 de febrero en pacientes menores de 13 años, sólo existen reportes y estimados en poblaciones de mayor edad ${ }^{22,33,34}$. En la tabla 6 se comparan las diferentes prevalencias de TEPT observadas en estudios chilenos. Se muestra la prevalencia de TEPT sin relación a un desastre en una población desde los 15 años en adelante con la escala DIS-III-R ${ }^{33,34}$, con un estudio realizado en adolescentes de 13 a 19 años luego del terremoto/maremoto del 27 de febrero de 2010 que aplicó la escala CPSS siete meses después del desastre en la Octava Región ${ }^{28}$ y los resultados obtenidos en el presente trabajo, evidenciándose que estos últimos muestran prevalencia significativamente más elevada en ambos sexos; además, este estudio muestra un mayor riesgo de TEPT en niños de menor edad. Estos resultados apoyan la evidencia respecto a que los niños de menor edad son una población de riesgo ${ }^{23,24,26}$ (concepto aún en discusión), aunque se debe considerar que ambos estudios evaluaron diferentes localidades, siendo el de Díaz et al. ${ }^{22}$, aplicado en una ciudad que sólo fue afectada por el terremoto y no por maremoto, lo que podría ser otro factor que influyó en las importantes diferencias.

Respecto a la prevalencia de TEPT según sexo, estos hallazgos concuerdan con la bibliografía, mostrando mayor riesgo de TEPT en mujeres ${ }^{22,24}$. Se hizo el análisis de los datos además según curso del niño, encontrándose resultados inversos a los del estudio de Liu et $\mathrm{al}^{25}$, donde observaron menor prevalencia en niños de tercer grado (edades equivalentes al curso chileno). Los actuales resultados son 
más acordes a la literatura, que documenta mayor riesgo de TEPT a menor edad y desarrollo cognitivo.

No se encontró diferencias significativas entre los grupos sintomáticos en relación a la edad, teniendo los niños de menor edad mayor puntaje en todos ellos. Tampoco se encontró relación entre TEPT y el tipo de familia en que el niño vive, lo que es acorde a la literatura ${ }^{26}$; sin embargo, llama la atención que, si bien las diferencias no son significativas, tienen menor puntaje en promedio los niños de familias monoparentales y quienes no viven con sus padres. Se requerirían estudios con mayor casuística para verificar esta tendencia, que podría relacionarse con la mayor exposición a síntomas de TEPT de los adultos cuidadores en los niños que viven con más adultos.

Una limitante de importancia del presente estudio es que, dada la edad de validación de la escala, no se aplicó a niños menores de 8 años, por lo que se desconoce lo ocurrido en dicho grupo etario. Otra limitación es que no se relacionó la sintomatología con el grado de daño personal o familiar de cada niño, que se conoce es un factor determinante en la presencia de TEPT; sin embargo, las consecuencias personales y materiales en la población estudiada fueron relativamente uniformes, sin que hubiera víctimas fatales en los habitantes de la localidad, y con un nivel de destrucción por el maremoto relevante y bastante similar en el poblado $^{35}$.

Este estudio se realizó sólo al finalizar una intervención con los niños de la localidad, en la cual se evaluó a quienes fueron derivados por los profesores, que fue un total de $33 \mathrm{ni}-$ ños, de los cuales sólo tres cumplían criterios para $\mathrm{TEPT}^{4}$. Se evidencia que los profesores detectaron menos niños sintomáticos, lo que está descrito que ocurre habitualmente en sintomatología internalizante ${ }^{36}$. Se sugiere ante otras intervenciones similares en desastres, efectuar un screening directamente con los niños a través de escalas validadas previo a la intervención, para evitar esta situación de subdiagnóstico, lo que además permitiría evaluar los resultados de la intervención con una aplicación posterior de la escala.

Finalmente, parecería necesario en suce- sivos estudios cambiar el foco sólo desde el TEPT al amplio rango de los efectos negativos de los eventos traumáticos, que incluyen otras patologías ${ }^{14} \mathrm{y}$, desde otra mirada, conocer los posibles efectos positivos, ya que se ha descrito la presencia en estas poblaciones del Crecimiento Postraumático, definido en 1996 por Calhoun y Tedeschi como los cambios psicológicos positivos resultantes de la lucha con circunstancias de vida altamente desafiantes ${ }^{37}$.

Se concluye la importancia de examinar directamente y brindar atención especializada a los niños más pequeños cuando son víctimas de desastres naturales, dado que un alto porcentaje de ellos podrá desarrollar un TEPT, encontrándose en mayor riesgo las mujeres y niños de menor edad. Se requeriría mayor investigación para explicar la vulnerabilidad específica de esta población estudiada, si ésta sólo se puede entender por el grado de destrucción del desastre o por otros factores, y si las múltiples intervenciones terapéuticas realizadas y el apoyo social han sido efectivos en disminuir la prevalencia y en prevenir las consecuencias a largo plazo del trastorno.

\section{Referencias}

1.- United States Geological Survey's: Historic World Earthquakes [Internet]. Última actualización 1 Nov 2012. Disponible en http://earthquake.usgs.gov/earthquakes/ world/historical_mag.php

2.- CEPAL: Terremoto en Chile: Una primera mirada al 10 de Marzo de 2010 [Internet]. Santiago: Publicación de las Naciones Unidas; 2010. Disponible en http://www. eclac.cl/desastres/publicaciones/sinsigla/xml/6/41476/ terremoto_CHILE.pdf

3.- Correa A, Abufhele M, Briceño AM, et al: Intervención de salud mental para niños expuestos al desastre en la comunidad de Iloca: Experiencia de la Unidad de Psiquiatría Infantil de Clínica Alemana. Rev Chil Psiquiatr Neurol Infanc Adolesc 2011; 22 (1): 92-101. Disponible en http://www.sopnia.com/boletines/Revista\%20SOPNIA\%202011-1.pdf

4.- Sommer K, Abufhele M, Briceño AM, et al: Implementación y Evaluación de la Intervención de Salud Mental para Niños Expuestos al Desastre en la Escuela de Iloca: Experiencia de la Unidad de Psiquiatría Infantil de Clínica Alemana de Santiago (en prensa). 
5.- Cohen RE: Lecciones aprendidas durante desastres naturales: 1970-2007. Rev. perú. med. exp. salud publica, 2008, 25(1), 109-117. Disponible en http://www.scielo. org.pe/pdf/rins/v25n1/a13v25n1.pdf

6.- American Psychiatric Association: Manual Diagnóstico y Estadístico de los Trastornos Mentales, DSM-IV TR. Washington; Ed. Masson, 2003.

7.- Bustos P, Rincón P, Aedo J: Validación Preliminar de la Escala Infantil de Síntomas del Trastorno de Estrés Postraumático (Child PTSD Symptom Scale, CPSS) en Niños/as y Adolescentes Víctimas de Violencia Sexual. Psykhe 2009; 18 (2): 113-26.

8.- Alisic E, Kleber R: Measuring Posttraumatic stress reactions in children: A preliminary validation of the children`s responses to trauma inventory. J Child Adol Trauma 2010; 3: 192-204.

9.- Eksi A, Braun K: Over-time changes in PTSD and depression among children surviving the 1999 Istanbul earthquake. Eur Child Adoles Psy 2009; 18: 384-91.

10.- Weil K, Florenzano R, Vitriol V, et al: Trauma infanto juvenil y psicopatología adulta: un estudio empírico. Rev Med Chile 2004; 132: 1499-504. Disponible en http://www.scielo.cl/scielo.php?pid=S003498872004001200007\&script=sci_arttext\&tlng=en

11.- Zahradnik M, Stewart SH, O'Connor RM, Stevens D, Ungar M, Wekerle C: Resilience Moderates the Relationship between Exposure to Violence and Posttraumatic Reexperiencing in Mi»kmaq Youth. Int J Ment Health 2010; 8 (2): 408-20.

12.- Michael T, Ehlers A, Halligan SL, Clark DM: Unwanted memories of assault: what intrusion characteristics are associated with PTSD? Behav Res Ther 2005; 43: 61328.

13.- Read JP, Brown PJ, Kahler CW: Substance use and posttraumatic stress disorders: symptom interplay and effects on outcome. Addict Behav 2004; 29: 1665-72.

14.- Coffey SF, Stasiewicz PR, Hughes PM, Brimo ML: Trauma-focused imaginal exposure for individuals with comorbid posttraumatic stress disorder and alcohol dependence: Revealing mechanisms of alcohol craving in a cue reactivity paradigm. Psychol Addict Behav 2006; 20: 425-35. Eksi A, Braun K. Over-time changes in PTSD and depression among children surviving the 1999 Istanbul earthquake. Eur Child Adoles Psy 2009; 18: 384-91.

15.- Blom M, Oberink R: The validity of the DSM-IV PTSD criteria in children and adolescents: A Review. [Internet] Clin Child Psychol Psychiatry 2012; doi:10.1177/1359104511426408.

16.- Montt ME, Hermosilla W: Trastorno de Estrés Postrau- mático en niños. Rev Chil Neuro-Psiquiatría 2001; 39 (2): 110-20.

17.- Bas P, Fernández M, Barbacil A, Gutiérrez C: Perspectivas actuales en el tratamiento del trastorno de estrés postraumático en la población infantil: Análisis bibliométrico. Clínica y Salud 2006; 17 (1): 69-89.

18.- Evans L, Oehler-Stinnett J: Children and natural disasters: A primer for school psychologist. School Psychol Int 2006; 27: 33-55.

19.- Salcioglu E, Basoglu M: Psychological effects of earthquakes in children: prospects for brief behavioral treatment. World J Pediatr 2008; 4 (3): 1-8.

20.- Şahin NH, Batıgün AD, Yılmaz B: Psychological symptoms of Turkish children and adolescents after the 1999 earthquake: exposure, gender, location, and time duration. J Trauma Stress 2007; 20: 335-45.

21.- Bal A, Jensen B: Post-traumatic stress disorder symptom clusters in Turkist child and adolescent trauma survivors. Eur Child Adoles Psy 2007; 16: 449-57.

22.- Díaz C, Quintana G, Vogel E: Síntomas de depresión, ansiedad y estrés post-traumático en adolescentes siete meses después del terremoto del 27 de febrero de 2010 en Chile. Terapia Psicológica 2010; 30 (1): 37-43.

23.- Piyasil V, et al: Psychiatric disorders in children at one year after the tsunami disaster in Thailand. Journal of The Medical Association of Thailand 2008; 91: 315-20.

24.- Margoob M, Wanj Z, Murtaza I, et al: Posttraumatic stress disorder: an overview. JK-Practitioner 2004; 11 (2): 88-94.

25.- Liu M, Wang L, Shi Z, Zhang Z, Zhang K, Shen J: Mental health problems among children one-year after Sichuan Earthquake in China: a follow-up study. PLoS One 2011; 23 (6): 2: e14706.

26.- Hinojos-Gallardo LC, Ruiz-Escalona L, Cisneros-Castolo M, Mireles-Vega E, Pando-Tarín GA, Bejarano-Marin $J M$ : Estrés postraumático en la población pediátrica atendida en el Hospital Infantil del estado de Chihuahua, México. Bol. Med. Hosp. Infant. Mex. 2011; 68 (4). Accedido en 08 Jul 2012. Disponible en http://www. scielo.org.mx/scielo.php?script=sci_arttext\&pid=S1665$11462011000400007 \& \operatorname{lng}=$ es\&nrm $=$ iso

27.- Dyregrov A, Yule W: A review of PTSD in children. Child and Adolescent Mental Health 2006; 11: 176-84.

28.- Foa E, Johnson K, Feeny N, Treadwell K: The Child PTSD Symptom Scale: A preliminary examination of its psychometric properties. J Clin Child Psychol 2001; 30: 376-84.

29.- Şahin NH, Batıgün AD, Yılmaz B: Psychological symptoms of Turkish children and adolescents after the 1999 earthquake: exposure, gender, location, and time 
duration. J Trauma Stress 2007; 20: 335-45.

30.- Goenjian AK, Pynoos RS, Steinberg AM, et al: Psychiatric comorbidity in children after the 1988 earthquake in Armenia. J Am Acad Child Adolesc. Psy 1995; 34: 1174-84

31.- Panadero S, Vázquez J, Rincón P, et al: Evaluación y reducción del impacto en la población afectada por el terremoto de Chile, en la comuna de Talcahuano [Internet]. Disponible en http://www.quintocongresocud.es/ pdf/501.pdf

32.- De Vries A, Kassam-Adams N, Cnaan A, Sherman-Slate E, Gallagher P, Winston F: Looking beyond the Physical Injury: Posttraumatic Stress Disorder in Children and Parents after Pediatric Traffic Injury. Pediatrics 1999; 104 (6): 1293-9.

33.- Vicente B, Rioseco P, Saldivia S, Torres S. Estudio chileno de prevalencia de patología psiquiátrica (DSM-III-R/ CIDI) (ECPP). Rev Med Chile 2002; 130: 527-36.

34.- Pérez-Benitez C, Vicente B, Zlotnick $C$, et al: Estudio epidemiológico de sucesos traumáticos, trastorno de estrés post-traumático y otros trastornos psiquiátricos en una muestra representativa de Chile. Salud Mental 2009; 32 (2): 145-53.

35.- El Mercurio Online. (06/Marzo/2010). Bachelet resalta "hazaña" de Carabinero en Iloca al evitar muertes producto del tsunami. [Internet] Disponible en http:// www.emol.com/noticias/nacional/2010/03/06/402076/ bachelet-resalta-hazana-de-carabineros-en-iloca-alevitar-muertes-producto-del-tsunami.html

36.- Reich W: Diagnostic Interview for Children and Adolescents (DICA). J Am Acad Child Adolesc Psy 2000; 39 (1) 59-66.

37.- Tedeschi R, Calhoun L: Posttraumatic Growth: Conceptual Foundations and Empirical Evidence. Psychol Inq 2004; 15 (1): 1-18. Disponible en http://data.psych.udel.edu/abelcher/Shared $\% 2520$ Documents/3\%2520Psychopathology\%2520(27)/ Tedeschi,\%2520Calhoun,\%25202004.pdf 